\title{
CJEM J J
}

MAY / MAl 2015 Vol. 17 Supplement 2

\section{CAEP/ACMU 2015 Scientific Abstracts}

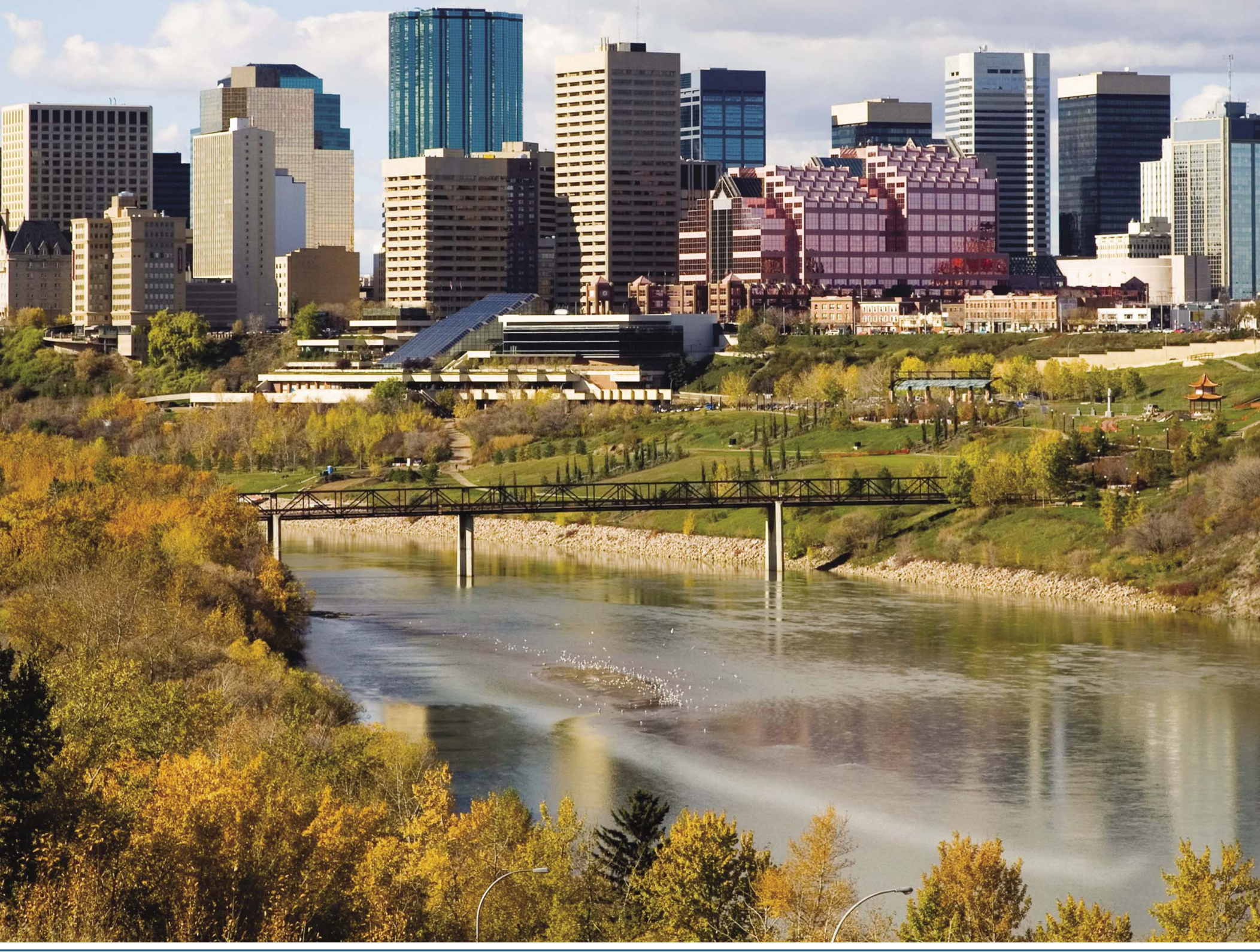

$5 \sqrt{4} / y_{1}^{2}$ 


\section{Canadian Journal of Emergency Medicine Editorial Board}

\section{Editor-in-Chief}

James Ducharme, MD

McMaster University, Canada

\section{Senior Associate Editors}

Riyad Abu-Laban, MD, MHSc

University of British Columbia, Canada

Eddy Lang, MD, CM

University of Calgary, Canada

Laurie J. Morrison, MD, MSc

University of Toronto, Canada

Marco Sivilotti, MD, MSc

Queen's University, Canada

\section{Managing Editor}

Andrea Schaffeler, BA BJourn

Pickering, Canada

\section{Assistant Managing Editor \\ Jennifer D. Artz, PhD \\ Ottawa, Canada}

\section{Editor Emeritus}

Grant Innes, MD

University of Calgary, Canada

\author{
Associate Editors \\ Steven Brooks, MD, MHSc \\ Queen's University, Canada \\ State of the Art \\ Garth Dickinson, MD \\ University of Ottawa, Canada \\ International EM \\ Jeff Eppler, MD \\ Kelowna General Hospital, Canada \\ Pharmacotherapy \\ Christopher M.B. Fernandes, MD \\ University of Western Ontario, Canada \\ ED Administration \\ Jeffrey Freeman, MD \\ University of Michigan, USA \\ Editorials / Radical Departure \\ Ran Goldman, MD \\ University of British Columbia, Canada \\ Pediatric EM \\ Niranjan Kissoon, MD \\ University of British Columbia, Canada \\ Pediatric EM \\ Jacques S. Lee, MD \\ University of Toronto, Canada \\ EM Advances \\ David Mann, MD \\ Powell River General Hospital, Canada \\ Community EM \\ Rohit Mohindra, MASc, MD \\ McGill University, Canada \\ Resident Issues \\ Heather Murray, MD, MSc \\ Queen's University, Canada \\ EM Advances
}

Roy Purssell, MD

University of British Columbia, Canada

Toxicology

Jonathan Sherbino, MD, MEd

St. Joseph's Hospital, Canada

Education

Brian Steinhart, MD

St. Michael's Hospital, Canada

Diagnostic Challenge

Ian G. Stiell, MD, MSc

University of Ottawa, Canada

EM Advances

Jo-Ann Talbot, MD

Dalhousie University, Canada

Journal Club

Andrew Worster, MD, MSc

McMaster University, Canada

Methodology: The Science of EM

Peter J. Zed, BSc(Pharm), PharmD

University of British Columbia, Canada

Pharmacotherapy

\section{Methodology Reviewers}

Andrew G. Day, MSc

Queen's University, Canada

Garth Hunte, MD, PhD

University of British Columbia, Canada

Rob Stenstrom, MD, PhD

University of British Columbia, Canada

\section{Editors at Large}

Paul Atkinson, MS, BCh, BAP, MA

Dalhousie University \& Saint John Regional Hospital, Canada

Alix Carter, MD, MPH

Dalhousie University, Canada

Stephen Choi, MD

The Ottawa Hospital, Canada

Jim Christenson, MD

University of British Columbia, Canada

Robert S. Green, MD

Dalhousie University, Canada

James Maskalyk, MD

St. Michael's Hospital, Canada

Andrew McRae, MD, PhD

University of Calgary, Canada

Jeffrey Perry, MD, MSc

University of Ottawa, Canada

Michael J. Schull, MD

Toronto, Canada

Andrew H. Travers, MD, MSc (Epidemiol)

Halifax, Canada

Suneel Upadhye, MC

Hamilton, Canada

\author{
Social Media Editors \\ Teresa Chan, MD \\ McMaster University, Canadaw \\ Rohit Mohindra, MASc, MD \\ McGill University, Canada
}




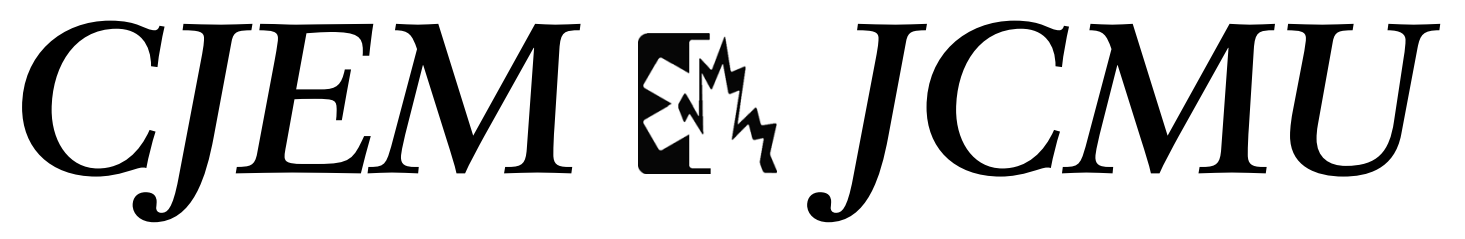

Vol. 17, No. S2

May 2015/

mai 2015

\section{Table of Contents • Table des matières}

Message from Research Committee Chair

CAEP/ACMU 2015 Research Awards

ABSTRACTS

Plenary Oral Presentations

Oral Presentations

Moderated Poster Presentations

S29

Poster Presentations

S54

INDEXES

Keyword Index

S89

Author Index 